\title{
Experimental Research for Chemical Flooding of Heavy Oil
}

\author{
Yanfu Pi \\ Key laboratory of enhanced oil and gas recovery of \\ ministry of education of china Petroleum Engineering, \\ Northeast Petroleum University \\ Daqing, China \\ Email: piyanfu@163.com
}

\author{
Yingjie Liu \\ Petroleum Engineering \\ Northeast Petroleum University \\ Daqing, China \\ E-mail:965265699@qq.com
}

\author{
Xiaofang Yang \\ Technical Service Center of Oil production \\ Petroleum Engineering Institute of Dagang Oilfield \\ Tianjin, China \\ Email: yeyxf@163.com
}

\begin{abstract}
Polymer flooding has already been the preferred development mode for the efficient development in offshore, aim at the conditions of the reservoirs in offshore, this paper has used two-dimensional physical model to conduct three tube parallel experiments to study the displacement efficiency of water flooding and polymer flooding, used real-time monitoring system of saturation to monitor the conditions of the use and swept for the heterogeneous reservoirs. The results showed that when the composite water cut reached 95\% during the water flooding, from the overall perspective,

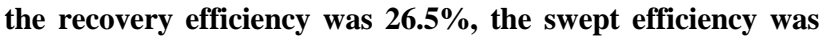
$48 \%$, the oil displacement efficiency was $55.2 \%$; the polymer was injected when the water cut was $70 \%$ during the stages of water flooding, from the overall perspective, the recovery efficiency was $36.2 \%$, the sweep efficiency was $65 \%$, the oil displacement efficiency was $\mathbf{5 9 . 4 \%}$. It could be seen from the curve of shunt index that the shunt index for the middle permeability layer during the stage of polymer flooding increased apparently, which turned out that the efficiency of expanding swept volume is better than the efficiency of enhancing displacement efficiency during the stage of polymer flooding.
\end{abstract}

Keywords-polymer flooding;offshore oilfield;recovery efficiency;swept efficiency;displacement efficiency.

\section{INTRODUCTION}

The sustained growth of the offshore oil production has become an important part of oil production capacity to replace, it is imperative to development the enhanced oil recovery technology for offshore reservoirs [1]. The chemical flooding technology has developed gradually towards the offshore reservoirs with the high risk whose reservoir conditions are complex, whose injection conditions are extremely hard [2-3]. Because of the complex reservoir conditions, high temperature, hypersalinity, high viscosity of crude oil and high expense cost of conducting chemical flooding in the offshore reservoirs [4-5], the practices of polymer flooding in land oilfield [6-8] show that the mechanical capture, the chemisorption and the function of retention of the polymer molecule during the process of the seepage for pore medium in the reservoirs can lead to that the permeability of the pore medium decreases, the flow resistance increases, and the higher resistance coefficient and residual resistance factor could be established in the high permeability layers, consequently, the oil recovery could be enhanced [9-10]. Polymer flooding as the most potential chemical flooding technology has been the preferred way of displacement after water flooding. Aim at the reservoir conditions and exploitation situation of typical block in offshore reservoirs, this paper used heterogeneous physical model which contained three different thickness layers to conduct the parallel physical simulation experiments, conducted comparison of displacement efficiency between water flooding and polymer flooding, and studied the dynamic seepage rules.

\section{THE SECTION OF EXPERIMENTS}

\section{A. Experimental Conditions and Schemes}

The experimental water is the simulated formation water of offshore oilfield whose salinity is 9374 $\mathrm{mg} / \mathrm{l}$; the experimental oil is the simulation oil which is mixed with crude oil and kerosene oil and whose viscosity is $70 \mathrm{mPa} \cdot \mathrm{s}$ when the temperature is $65^{\circ} \mathrm{C}$; the physical models which are used in the experiments are heterogeneous cores whose saturation can be monitored and which are cemented by quartz sand and epoxy resin, whose specification is $300 \mathrm{~mm} \times 300 \mathrm{~mm} \times$ $30 \mathrm{~mm}$ and the electrodes are laid in the different permeable layers and permeability of each layer is respectively $4800 \times 10-3 \mu \mathrm{m} 2, \quad 2200 \times 10-3 \mu \mathrm{m} 2, \quad 500 \times 10-$ $3 \mu \mathrm{m} 2$; the polymer which is used in the experiments in the stage of polymer flooding is AP-P4 hydrophobic associative polymer whose concentration is $2000 \mathrm{mg} / \mathrm{l}$ and the viscosity is $42 \mathrm{mPa} \cdot \mathrm{s}$; The experiments are conducted under the temperature condition of $65{ }^{\circ} \mathrm{C}$; the speed of injection in the experiments is $3 \mathrm{ml} / \mathrm{min}$. The experiments adopt the form of parallel and the experimental sketch map is as shown in Figure 1.The real-time monitoring system of saturation can monitor the conditions of the oil saturation for every monitoring point, the condition of swept and use 
are judged by whether the saturation values of the monitoring points change or not in the experiments. There are two experiments, one (Scheme1) is that water flooding is conducted until the water cut is $95 \%$, the other (Scheme2) is that when the water cut is $70 \%$ during the water flooding, polymer flooding are conducted, and the injection of polymer is 0.3 times of the pore volume injected, and then further water flooding is conducted until the water cut is $95 \%$.

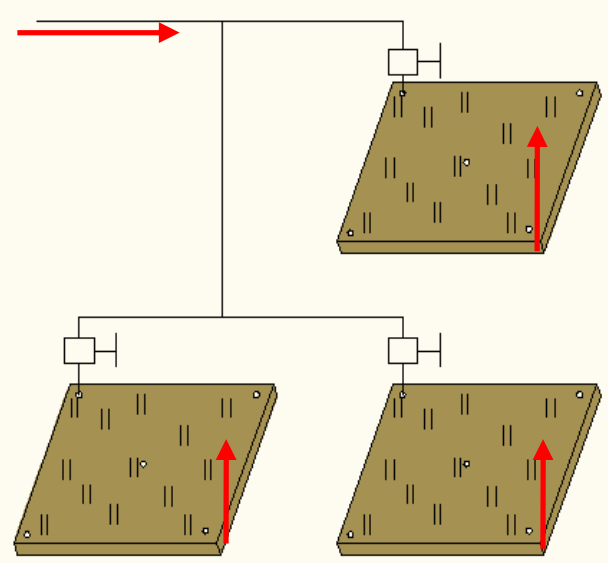

FigureI the sketch map of parallel displacement experiments

\section{B. Experiment Process}

1. Evacuate the cores, saturate water for the cores, then saturate oil for the cores saturated water;

2. Weld for the eletrodes;

3. Conduct the displacement experiments according to the exoeriment schemes, and record liquid producing capacity, water production and the pressure at every time quantum, and acquire the values of resistancs.

\section{EXPERIMENTAL RESULTS AND ANALYSIS}

\section{A. Experimental Results and Analysis or Every Stages}

The experimental results and analysis for every stage is as shown as TABLE I.

TABLE I. THE DEGREES OF RESERVE RECOVERY IN THE DIFFERENT DISPLACEMENT STAGES

\begin{tabular}{|c|c|c|c|c|}
\hline & \multicolumn{3}{|c|}{$\begin{array}{l}\text { The degrees of reserve } \\
\text { recovery for layers in } \\
\text { different permeability } \\
\text { layer }(\%)\end{array}$} & \multirow[t]{2}{*}{$\begin{array}{c}\text { Overall } \\
\text { recovery } \\
\text { efficiency } \\
(\%)\end{array}$} \\
\hline & Low & Middle & High & \\
\hline $\begin{array}{l}\text { Water flooding to } \\
\text { the water cut is } 70 \%\end{array}$ & 4.5 & 15.8 & 23.6 & 18.0 \\
\hline $\begin{array}{l}\text { Water flooding to } \\
\text { the water cut is } 90 \%\end{array}$ & 4.5 & 17.4 & 40.2 & 26.5 \\
\hline $\begin{array}{l}\text { Polymer flooding } \\
\text { (containing the } \\
\text { further water } \\
\text { flooding ) }\end{array}$ & 5.7 & 16.2 & 25.2 & 36.2 \\
\hline $\begin{array}{l}\text { Overall recovery } \\
\text { efficiency (in the } \\
\text { overall model) }\end{array}$ & 10.2 & 32.0 & 48.8 & \\
\hline
\end{tabular}

It can be seen from the Table1 that the overall recovery efficiency of the model for the Scheme 1 is $26.5 \%$, when the water cut is from $70 \%$ to $95 \%$ during the water flooding, the low permeability layer hasn't been employed, the degrees of reserve recovery of middle permeability layer is $1.6 \%$, and high permeability layer's is $16.6 \%$. The overall recovery efficiency of the model for the Scheme 1 is $36.2 \%$, from the beginning of polymer flooding to the end of further water flooding, the degrees of reserve recovery of high permeability layer is $8.6 \%$, low permeability layer's is $5.7 \%$, and middle permeability layer's is $14.6 \%$, the overall recovery efficiency of the model has increased by $9.7 \%$, so it mainly enhances the degrees of reserve recovery of middle permeability layer during the stage of polymer flooding.

TABLE II. RESULTS OF SWEEP EFFCIENCY AND OIL

DISPLACEMENT EFFICIENCY WITH DIFFERENT SCHEME

\begin{tabular}{|c|c|c|c|c|c|}
\hline Parameters & Scheme & \multicolumn{3}{|c|}{ permeability layer } & overall \\
\cline { 2 - 6 } & & Low & Middle & High & \\
\hline $\begin{array}{c}\text { Swept efficiency } \\
\text { (\%) }\end{array}$ & 1 & 13.0 & 41.0 & 90.1 & 48.0 \\
\cline { 2 - 6 } & 2 & 37.5 & 67.0 & 90.5 & 65.0 \\
\hline $\begin{array}{c}\text { Displacement } \\
\text { efficiency (\%) }\end{array}$ & 1 & 34.6 & 42.4 & 44.6 & 55.2 \\
\cline { 2 - 6 } & 2 & 40.3 & 53.7 & 64.2 & 59.4 \\
\hline
\end{tabular}

The displacement situation can be seen from calculating and statistics for every scheme as shown as Table 2, it can be seen from the swept efficiency of every layer that the high permeability layer is mainly employed during the stage of water flooding, and the swept efficiency are high which is $0.901 \%$. In the Scheme2, the swept efficiency of the low, middle, and high permeability layers are increasing based on the water flooding at the end of displacement, but because the high permeability layer has already been employed on the whole at the stage of water flooding, its swept efficiency only enhances by $0.4 \%$; and the middle and low permeability layers' enhance by $20 \%$, this experiment result shows that the polymer mainly enlarges the swept efficiency of middle and low permeability layers, and enhances the displacement efficiency for the high permeability layer.

\section{B. The Dymamic Recovery Curve of Every Scheme and} Analysis.

The dynamic recovery curves contain the stage of water flooding and the stage of polymer flooding.

1. The dynamic recovery curve of every scheme and analysis for the water flooding stage which is as shown as Figure II. 


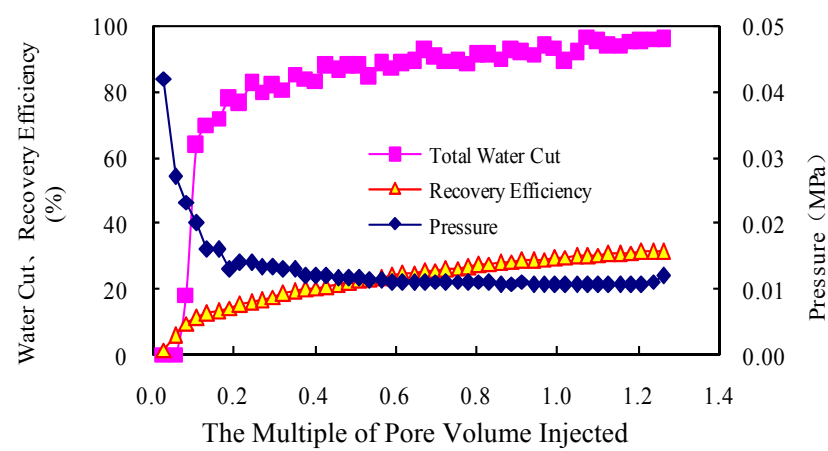

Figure II. Relation curve between pore volume injected and water cut, recovery and pressure for the stage of water flooding

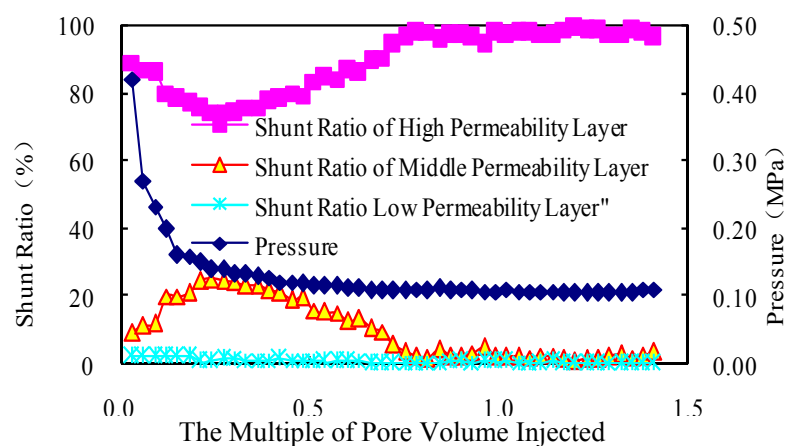

Figure III. Relation curve between the multiple of pore volume injected and shunt ratio, pressure for the stage of water flooding

It can be seen from Figure II and Figure III that the pressure increases suddenly at the beginning of water flooding, and decreases quickly with the increase of injected water volume. During the whole progress, water cut increases quickly at the beginning, and it starts to increase slowly when it reaches to $70 \%$, at the moment injection pressure decreases slowly and closes to stable gradually.

It can be seen from the shunt ratio curve of every layer at the water flooding stage that absorption amount is very high in the high permeability layer, which is mainly because that filtrational resistance of the high permeability layer is small and the liquid is easy to enter into, and the thickness in this layer is higher and the pore volume accounts for $51 \%$ of the total pore volume; the middle and low permeability layers have reached better efficiency of shunt and profile control.

2. The dynamic recovery curve of every scheme and analysis for the stage of water flooding and polymer flooding as shown as Figure IV.

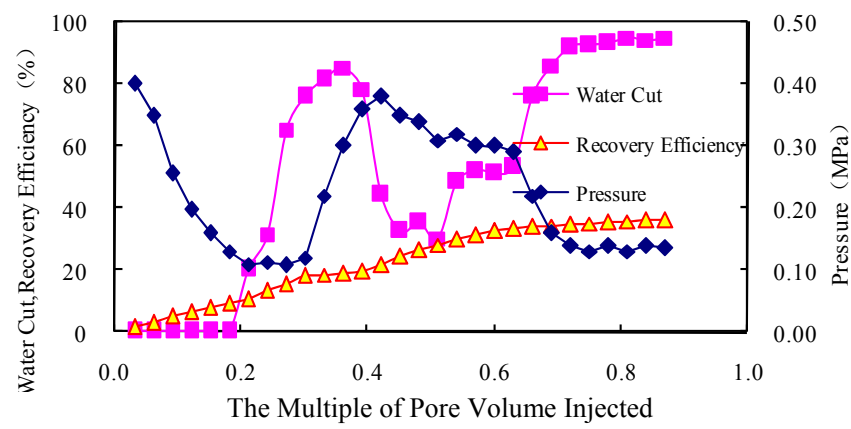

Figure IV Relation curve between pore volume injected and water cut, recovery and pressure for the stage of water flooding and polymer flooding

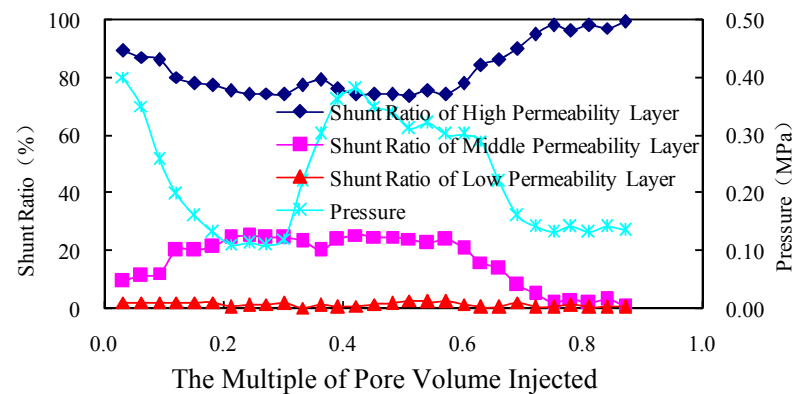

FigureV Relation curve between pore volume injected and shunt ratio, pressure for the stage of water flooding and polymer flooding

It can be seen from Figure $\mathrm{V}$ that polymer starts to be injected when the composite water cut reaches at $70 \%$, the pressure starts to increase after the polymer is injected, but composite water cut increases first and then decreases, the hysteresis effect of the polymer has appeared. The pressure begins to increase with the injection of polymer, the shunt ratio of high permeability layer starts to decrease, but middle and low's increase gradually, and the rising trend of low permeability layer is more apparent than water flooding. During the stage of further water flooding, the pressure starts to appear downtrend, it decreases quickly after the mainstream channel of high permeability is opened completely, and it fluctuates stably around a certain value, at the moment, the shunt ratio of high permeability layer has reached at above $90 \%$ and the middle and low's are very small, and middle and low permeability layers can't continue to be exploited because the liquid entering into is rarely.

\section{CONCLUSIONS}

1. The overall swept efficiency is $48 \%$ when the total water cut is $95 \%$ during the water flooding, polymer starts to be injected when the water cut reaches at $70 \%$, and overall swept efficiency is $65 \%$, the shunt ratio of middle permeability layer increases apparently during the polymer flooding, which turns out that the efficiency of enlarging swept volume for polymer is apparent.

2 . The overall recovery efficiency is $26.5 \%$ when the total water cut is $95 \%$ during the water flooding, polymer starts to be injected when the water cut reaches at $70 \%$, and overall recovery efficiency is $36.2 \%$, which turns out that polymer injected whose volume is 0.3 times than pore volume can enhance the overall recovery efficiency by $9.7 \%$, and the displacement efficiency of polymer flooding is apparent.

\section{ACKNOWLEDGMENT}

The research work was joint funding between the national major projects "Reservoir Research for Enhanced Oil Recovery Technology after Polymer Flooding in Offshore Oilfields"(2011ZX05024-004-14) and the national natural and scientific foundation of China"Research in micro pore number dynamics mechanism of crude oil activation conditions" No. 51104030 . 


\section{REFERENCES}

[1] Chuanfei Wang, "Reservoir Potential Evaluation and Research of Enhancing Oil Recovery of Chemical Flooding in Offshore Oilfields-taking Suizhong 36-1 Reservoir as Example," China University of Petroleum, 2010, 5-8.

[2] Shanshan Jiang, Junru Yang, Jiefu Sun, Xiansong Zhang, Haijiang Wang, Engao Tang, "Injection Time Study and Field Application of Polymer in Offshore Oilfields," Offshore Oil, vol.29, Sep.2009, pp.37- 38, doi:10.3969.

[3] Fengjiu Zhang, Wei Jiang, Fujie Sun, and Shouwei Zhou, "Key Technology Research and Field Test of Polymer Flooding for Heavy Oil in Offshore Oilfields," Chinese Engineering Science, vol.13, May.2011, pp. 28-29, doi:10.3969.

[4] Chengqiang Shu, "Research of Guiding Field Test of Enhancing Oil Recovery for Association Polymer in J3 Wellblock of Bohai Oilfield," Southwest Petroleum Institute, 2005.

[5] Shouwei Zhou, Ming Han, Wentao Xiang, Jian Zhang, Wei Zhou, Wei Jiang, "The Technology Research and Application of Enhancing Oil Recovery with Polymer Flooding in Bohai
Oilfield, " China Offshore Oil and Gas, vol.18, Dec.2006, pp. 386-387, doi:10.3969.

[6] Demin Wang, Heng Liu, Jingang Niu, and FuMing Chen, "Application Results and Understanding of Several Problems of Industrial Scale Polymer Flooding in Daqing Oil Field, ” SPE 50928, 1998: 477-481.

[7] Zhenhua Zhang, Jiecheng Cheng, and Lin Li, "The Pilot Test Technology of Polymer Flooding, " Beijing: Petroleum Industry Press, 1996.

[8] Liu Li. The Distribution and Measures for Potential Exploitation of Remaining Oil After Polymer Flooding. Daqing: Daqing Petroleum Institute, 2005.

[9] Xinmin Zhang, Yongjun Guo, Rusen Feng, "The Performance Study of Binary Compound System Which Suits for Suizhong 36-1 Reservoir in Bohai Oilfield, "China Offshore Oil and Gas, 2007,19 (1) : 30 - 34 .

[10] Lanlei Guo, "The laws of adsorbtion retention and the research of performance change for polymer. Oil and natural gas chemical industry, Dec.2011, vol.40, pp.587-589, doi:10. 3969. 